\title{
Trabajo Social, descolonización de las políticas públicas y saberes no hegemónicos
}

\author{
Francisca Gómez Lechaptois \\ Pontificia Universidad Católica de Chile (UC) \\ y Universidad Alberto Hurtado (UAH)
}

Trabajo Social, descolonización de las políticas públicas y saberes no hegemónicos

Resumen: El presente artículo busca develar la primacía de la racionalidad cientificista moderna que reduce y limita la acción política pública, así como mostrar la importancia que tiene la recuperación y visibilización de aquellos saberes y/o formas de conocimiento no hegemónicos que han sido históricamente excluidos del proceso decisorio de las policies. Para ello, aborda la noción de "políticas públicas colonizadoras" a las que subyace normativamente una idea hegemónica de desarrollo, y plausibiliza la idea de "descolonización de las políticas públicas" a través de la recuperación y visibilización de formas de conocimiento que incluyen realidades suprimidas, marginadas y/o excluidas. Asimismo, releva el aporte que el pensamiento latinoamericano puede tener para este proceso de descolonización e identifica algunos desafíos concretos que emergen para el Trabajo Social desde su vinculación con la perspectiva descolonizadora en las políticas públicas.

Palabras clave: Políticas públicas. Colonización. Descolonización. Pensamiento latinoamericano. Saberes no hegemónicos.

Trabalho social, descolonização das políticas públicas e saberes não hegemónicos

Resumo: O presente artigo busca revelar a primazia da racionalidade cientificista moderna que reduz e limita a ação política pública, e também, mostrar a importância que tem a recuperação e a visibilidade dos saberes e/ou formas de conhecimento não hegemónicos que foram historicamente excluídos do processo decisório das policies. Para tanto, se aborda a noção de "políticas públicas colonizadoras" para as quais subjaz normativamente uma ideia hegemónica de desenvolvimento e possibilita uma ideia de "descolonização das políticas públicas" a través da recuperação e a visibilidade das formas de conhecimento que incluem realidades suprimidas, marginadas e/ou excluídas. Assim, se releva o aporte que o pensamento latino-americano poderia ter para este processo de descolonização e se identificam alguns desafios concretos que emergem para o Trabalho Social a partir da sua vinculação com a perspectiva descolonizadora nas políticas públicas.

Palavras-chave: Políticas públicas. Colonização. Descolonização. Pensamento latino-americano. Saberes não hegemónicos.

\section{Social Work, Decolonization of Non-Hegemonic Public Polices and Knowledge}

Abstract: This article reveals the primacy of the modern scientistic rationality that reduces and limits public political action, and shows the importance of the revival and visibilization of non hegemonic forms of knowledge that have been historically excluded from decision making processes involving public policies. It looks at the concept of "colonizing public policies" those that normatively support a hegemonic idea of development, and affirms the idea of a "decolonization of public policies" through the recovery and visualization of the forms of knowledge that include suppressed realities, which are marginalized and or excluded. It thus reveals the support that Latin American thinking can have for this decolonization process and identifies some concrete challenges that emerge for Social Work from its connection with the decolonizing perspective of public policies.

Keywords: Public policies. Colonization. Decolonization. Latin American thinking. Non-hegemonic knowledge. 
De los beduinos de los desiertos de Medio Oriente: Habla el rico y muchos lo aprueban y encuentran elocuente su hablar sin sentido;

[2] habla el pobre con acierto y no le hacen caso; Habla el rico y lo escuchan en silencio, y ponen por las nubes su talento; Habla el pobre y dicen: ¿quién es éste?, y si cae encima lo empujan (DUSSEL, 2004, p. 170).

\section{Introducción}

No sólo en Chile sino en diversos lugares del mundo, asistimos hoy a un crecimiento en las demandas por participación en las decisiones que se toman sobre nuestras vidas como sujetos individuales y colectivos. Los Estados están progresivamente siendo presionados por romper o al menos flexibilizar sus estructuras jerárquicas, e incorporar a otros actores que hasta el momento no estaban siendo incorporados en la toma de decisiones públicas. Esto obliga a repensar las relaciones entre Estado y sociedad y obliga a repensar también uno de los principales mecanismos, formas o instrumentos de acción del Estado sobre lo público: las políticas públicas. Pero sucede que en América Latina, tanto el Estado como las políticas públicas han sido históricamente pensados desde referentes teóricos que provienen de países distintos a los nuestros, particularmente desde perspectivas anglosajonas. De ahí la afirmación de Roth Deubel (2010) de que existe una escasa contribución latinoamericana al desarrollo de marcos y teorías para el análisis de la acción pública. Esto tendría relación con la primacía de un pensamiento eurocéntrico ${ }^{1}$ que se instala en nuestro continente a partir de los procesos de conquista y colonización y que se perpetúa hasta nuestros días.

Emerge entonces para quienes desarrollan investigación y/o intervención en el ámbito de las políticas públicas en América Latina, en este caso particular para las y los trabajadores sociales, el desafío de analizar dicha hegemonía, develando sus supuestos y proponiendo alternativas de superación, o al menos de diálogo. Una posibilidad está en contraponer a los análisis tradicionales de las políticas públicas que concentran su mirada en aspectos técnicos de la misma, análisis críticos que pongan en cuestión las bases normativas de dichas construcciones (GÓMEZ LECHAPTOIS, 2013; MIRANDA, 2011). Otra posibilidad es la de poner en cuestión la hegemonía de los saberes y/o formas de conocimiento que se juegan en las políticas públicas y que obedecen a una tradición colonizadora que califica como inferiores todo tipo de conocimiento distinto al conocimiento científico. Porque, como señalan Dávila y Soto (2011, p. 11), "el estudio de las políticas públicas ha implicado el estudio de las acciones de los gobiernos y de los Estados, así como el contexto institucional y la interacción de los jugadores existentes en el sistema político de cada realidad nacional", pero, podríamos agregar, no ha profundizado en las formas en que se incorporan e interactúan (o no) los diversos saberes y/o formas de conocimiento.

Es en esta línea que se desarrollará el presente artículoº, intentando, por una parte, develar la primacía de un tipo de racionalidad hegemónica que reduce y limita la acción política pública, convirtiéndola en una "política pública colonizadora", y, por otra parte, mostrar la importancia que tiene la recuperación y visibilización de aquellos saberes y/o formas de conocimiento no hegemónicos que han sido históricamente excluidos del proceso decisorio de las policies. Con este fin, se aborda primero la noción de "políticas públicas colonizadoras" a las que subyace normativamente una idea hegemónica de desarrollo que da continuidad a la imposición de una racionalidad cientificista moderna, para, en segundo término, plausibilizar la idea de "descolonización de las políticas públicas" a través de la recuperación y visibilización de formas de conocimiento que incluyen realidades suprimidas, marginadas y/o excluidas. En tercer lugar, se aborda el aporte que el pensamiento latinoamericano puede tener para este proceso de descolonización, brindando para ello el ejemplo del Buen $V_{i v i r}{ }^{3}$, y se finaliza identificando algunos desafíos concretos que emergen para el Trabajo Social desde su vinculación con la perspectiva descolonizadora en las políticas públicas.

\section{Políticas públicas colonizadoras}

Es plausible hablar de "políticas públicas colonizadoras" si se comprende que las acciones políticas públicas implementadas en el continente latinoamericano se han sustentado históricamente en la noción de desarrollo como horizonte normativo, la cual, junto a otras figuras como civilización y progreso, se afirma en un trasfondo filosófico colonizador que acompaña el tiempo de conquista y colonización del territorio y que se perpetúa en el Estado moderno y en las acciones políticas públicas que desde él se ejecutan. Este trasfondo 
filosófico se caracteriza por cargar con una visión del Otro como inferior, como aquel que es "bárbaro" y que por tanto "debe ser" civilizado por aquél en quien, en la idea hegeliana, se ha encarnado el Espíritu Absoluto. En figuras tempranas como Juan Ginés de Sepúlveda (SANTOS, 2010) se encarnan estos valores que justifican la invasión española y la posterior expansión de la colonialidad del poder (QUIJANO, 1992) en los distintos ámbitos de la vida del continente.

A esta filosofía de carácter colonizador, se contrapone la filosofía colonizada, aquella donde el trabajo filosófico se reduce a hacer hermenéutica de obras ajenas (SANTOS, 2010). En esta lógica, podríamos afirmar que las políticas públicas siguen un camino similar, considerando que el Estado moderno, impulsor y creador de las mismas, se construye teniendo como referente central el modelo europeo. A pesar de la fructífera obra de Martí (2005) y de la fuerza de sus planteamientos "nuestroamericanos", y a pesar, en el caso chileno, de ideas como las de Bilbao (1856) y su Iniciativa de la América, bajo la figura del colonialismo interno (GONZÁLEZ CASANOVA, 2006), las elites gobernantes del continente se forman en base a su afán de tratar de asemejarse a Europa, sin preocupación por la producción de conocimiento autóctono (TUSSIE; DECIANCIO, 2011). De ahí la crítica del pensador cubano a aquellos "incapaces" que "quieren regir pueblos originales, de composición singular y violenta, con leyes heredadas de cuatro siglos de práctica libre en los Estados Unidos, de diecinueve siglos de monarquía en Francia” (MARTÍ, 2005, p. 32).

Europa y luego Estados Unidos toman la figura de "tutores morales" (JOBERT, 2004) de la población colonizada que debía transitar desde la barbarie a la civilización, desde el retraso al progreso, desde el subdesarrollo al desarrollo ${ }^{4}$. Las ideas que orientan la construcción y consolidación de los Estados en las nuevas naciones, dicen de bases filosóficas que justifican dicho rol al sostenerse en la superioridad europea (y estadounidense), de esta forma, el Estado moderno da continuidad al proceso colonizador que en teoría se había detenido con la formación de las nuevas repúblicas (QUIJANO, 2000; DUSSEL, 2006; GONZÁLEZ CASANOVA, 2006), y lo hace a través de acciones públicas que permiten la concreción de esta razón política "colonizada" (y colonizadora) en el nivel de lo factible (DUSSEL, 2001), es decir, a través de las políticas públicas.

Esta razón política colonizada representa un particular tipo de racionalidad, que corresponde a la racionalidad instrumental y que sostiene mayoritariamente las acciones políticas públicas y los modelos de análisis a ellas referidas. Ello se evidencia en modelos de análisis de políticas públicas predominantes en América Latina, como es el modelo racional, el cual tiene como objetivo el uso del análisis científico para solucionar problemas públicos, optimizar recursos disponibles y garantizar resultados políticos a partir de la acción planificada definida por grupos de expertos (CHAQUÉS BONAFONT, 2004). En este modelo el protagonismo de la razón corresponde más bien al de la ciencia y particularmente a su vertiente empirista; dicho desde Kusch (1999), en él existe una especie de obsesión por la racionalidad-instrumental, que no permite ver cualquier otra posibilidad. Por tanto, todo conocimiento distinto a su vertiente científico-empirista queda subordinado, invisibilizado y/o excluido.

La primacía de la racionalidad tecnocientífica implica a su vez la concentración de la toma de decisiones en grupos de expertos que cuentan con el conocimiento "adecuado". En el modelo racional se presupone la existencia de un decisor racional que es capaz de definir los objetivos y preferencias sobre un problema público concreto, siendo los grupos de expertos los que pueden definir los programas de acción gubernamental de forma objetiva y eficiente. En este contexto, cobra sentido la idea de Chaqués Bonafont (2004, p. 8) de que lo único que deben hacer los Estados "es dejarse asesorar por estos grupos de expertos-científicos, e intentar que la política (la deliberación y el debate) ocupe el menor espacio posible en el proceso".

La unicidad del método genera además la homogeneización de las aproximaciones a los problemas públicos, entendiendo la política pública como una "receta" aplicable a los distintos contextos sociales, políticos, económicos, culturales etc. Al respecto, en un sentido más amplio, Osman (2002, p. 37, traducción del autora) dirá que "la mayoría de las teorías del policymaking han sido derivadas de estudios de sociedades industrializadas desarrolladas que, en la mayoría de los casos, son consideradas insuficientes para explicar las políticas de los países en desarrollo debido a las variaciones contextuales"; mientras, algunos autores latinoamericanos "se preguntan hasta qué punto 'viaja' o no el enfoque de políticas públicas a distintos contextos políticos, culturales, históricos, económicos, diferentes a los teorizados por el modelo norteamericano” (DÁVILA; SOTO, 2011, p. 15).

Cuando hablamos entonces de políticas públicas colonizadoras en América Latina lo hacemos considerando que a ellas subyace normativamente una idea hegemónica de desarrollo que da continuidad a la imposición de una racionalidad cientificista moderna. Racionalidad que coincide con aquella que predomina en modelos hegemónicos de análisis de políticas públicas - evidenciado en este caso en el modelo racional -, y que desconoce otras formas de estar y ser en el mundo, como son por ejemplo, las cosmovisiones indígenas, afrodescendientes, populares, entre otras. En la misma lógica, las políticas públicas colonizadoras perpetúan el patrón de poder vigente basado en una estrecha forma de racionalidad que se pregunta por el cómo más que por los "porqués", lo que lleva a considerar el desarrollo como una cuestión exclusivamente técnica, y sobre la cual sólo los "expertos" pueden opinar. 


\section{La descolonización de las políticas públicas y los saberes no hegemónicos}

De lo anterior surge la pregunta sobre la plausibilidad de pensar en "políticas públicas descolonizadoras", o de hablar, más bien, de la "descolonización de las políticas públicas". En el sentido y ámbito en el que venimos trabajando, la descolonización implicaría reconocer primero que la comprensión del mundo es mucho más amplia que la comprensión occidental (e instrumental) del mundo, y segundo que la diversidad del mundo es infinita e incluye distintos modos de ser, pensar y sentir (SANTOS, 2009). Lo cual implica a su vez la recuperación de formas de conocimiento que incluyan realidades suprimidas, silenciadas o marginadas.

La descolonización en el ámbito de las políticas públicas significaría entonces la búsqueda de conocimientos y criterios de validez que otorguen visibilidad y den credibilidad a los saberes y prácticas ${ }^{5}$ de los grupos sociales que han sido históricamente invisibilizados, excluidos, oprimidos por prácticas colonizadoras. Esta idea nos lleva a considerar la importancia que tiene el lugar de enunciación (SANTOS, 2010), es decir, el pensar la política pública como una acción situada. Esta idea anticipaba Martí (2005, p. 33) cuando decía que "el buen gobernante en América no es el que sabe cómo se gobierna el alemán o el francés, sino el que sabe con qué elementos está hecho su país". Desde aquí, volvemos a los planteamientos de Osman (2002) y algunos autores latinoamericanos (DÁVILA; SOTO, 2011) que critican el hecho de que la formulación de las políticas públicas se derive de estudios de sociedades que aparecen como insuficientes para explicar el propio contexto. García Márquez (1982) describe bellamente esta idea en el discurso de aceptación del Premio Nobel de Literatura: "el desafío mayor para nosotros ha sido la insuficiencia de los recursos convencionales para hacer creíble nuestra vida".

Porque la ciencia moderna, protagonista y fiel acompañante de la noción hegemónica de desarrollo, consideró superficial y falso todas las formas de conocimiento que no correspondiesen a los criterios epistemológicos establecidos por la ciencia para sí misma; siendo que, como hemos visto, no es "natural" la primacía de la forma de conocer europea (y/o estadounidense), sino que ella responde a un proceso histórico particular que ha sido naturalizado y perpetuado en el tiempo.

Santos (2011, p. 36) dirá que no hay justicia social sin justicia cognitiva, y "la lucha por la justicia cognitiva no tendrá éxito si se sustenta únicamente en la idea de una distribución más equilibrada del conocimiento científico". Bajo la idea de la ecologia de saberes, este autor releva la idea de que no hay ignorancia o conocimiento en general, sino que todas las prácticas implican más de una forma de conocimiento, y por ello de ignorancia. Lo que sucede es que la moderna sociedad capitalista favorece prácticas en que prima el conocimiento científico. Es decir, no se trata - solo - de mejorar el acceso al conocimiento científico, sino de validar otros conocimientos que, en el caso de las políticas públicas se encuentran en determinados actores del proceso como son, por ejemplo, los llamados "beneficiarios" y que no necesariamente han sido formados en esta lógica de conocimiento. Se trata entonces de dar cuenta de la in-comunicación silenciada de los excluidos (DUSSEL, 2004), de aquellas voces históricamente invisibilizadas y marginadas por la racionalidad científica dominante en las policies ${ }^{6}$, de aquellos conocimien-

... poner en cuestión la hegemonía de los saberes y/o formas de conocimiento que se juegan en las políticas públicas y que obedecen a una tradición colonizadora que califica como inferiores todo tipo de conocimiento distinto al conocimiento científico...

tos que han sufrido de "violencia epistemológica" y la consecuente destrucción de sus prácticas sociales y descalificación de sus saberes y agentes sociales (SANTOS, 2009).

En este marco, surgen importantes preguntas que buscan dar cuerpo y sentido a la posibilidad de romper con esta in-comunicación silenciada en el ámbito de las políticas públicas ${ }^{7}$. Preguntas como: ¿cuáles serían los saberes, conocimientos, prácticas discursivas no hegemónicas, y sus concomitantes relaciones con las formas de racionalidad hegemónica en el mundo de lo público? ¿De qué manera se (des)encuentran estas diversas expresiones del logos (SALAZAR, 1968; ZEA, 1976)? ¿Emerge de ello una mezcla nueva, un híbrido? ¿O es más bien la idea de un tercero incluido, en que la generación de diálogos interculturales o pluriculturales no decanten en construcciones mezcladas sino que contengan la tensión entre opuestos, permitiendo su convivencia y la fertilidad que de ella emerge? (RIVERA CUSICANQUI, 2010; KUSCH, 2000). ¿De qué manera el contexto histórico sobredetermina y/o debería sobredeterminar las particularidades de las políticas públicas en América Latina? (DÁVILA; SOTO, 2011). ¿De qué manera conjurar esa obsesión por la racionalidad de las elites en América Latina (KUSCH, 1999), que les impide ver otras posibilidades? ¿Cómo puede la política pública o el implementador de la política, mantener vivo lo mejor de 
la cultura occidental (reflejado en la política) y, al mismo tiempo, reconocer el valor de la diversidad del mundo del "otro", diverso culturalmente? ¿Puede constituirse la política pública en una zona de contacto privilegiada, es decir, un campo social donde diferentes mundos de vida normativos, prácticas y conocimientos se encuentran, chocan e interactúan? (SANTOS, 2009). Todas estas preguntas ponen en juego las condiciones de posibilidad para pensar una política pública que no monopolice el saber (científico) sino que se construya en interacción con saberes no hegemónicos, no expertos o legos (CALLON, 1999), debilitando de esta forma la estructura jerárquica que prima en la política pública.

\section{El aporte del pensamiento latinoamericano a la descolonización de las políticas públicas}

Hemos mencionado que la política pública se ha construido mayoritariamente sobre teorías y perspectivas que desconocen la importancia del lugar de enunciación. De ahí que en esta discusión se torne relevante hacer alusión a aquellas teorías que han sido pensadas "en" y "desde" el propio contexto latinoamericano y que podrían contribuir - directa o indirectamente - al proceso de descolonización de las políticas públicas. Estamos haciendo referencia a la tradición del "pensamiento latinoamericano" que, tal como lo muestran Zea (1976), Devés (2000) y Saladino (2009), tiene una importante trayectoria en términos de producción de conocimiento. Este pensamiento se ha expresado en diversas búsquedas de "lo latinoamericano": la búsqueda por la cultura latinoamericana (MORANDÉ, 1984), la identidad latinoamericana (LARRAÍN, 1996), la filosofía latinoamericana (ZEA, 1976; SALAZAR, 1968), la teología latinoamericana (GUTIÉRREZ, 1971; SEGUNDO, 1978; SOBRINO, 1991); y se continúa desarrollando hasta nuestros días.

Importa precisar que la apelación al pensamiento latinoamericano en la esfera de las policies, no conlleva la negación de las tradiciones hegemónicas ni la esencialización de las perspectivas latinoamericanas - cayendo en uno de los dos polos del debate universalismo/particularismo -, sino que se trata de observar los puntos de diálogo que entre ellas puede haber. De esta forma, se intenta evitar los etnocentrismos, ya sea en su expresión eurocéntrica (WALLERSTEIN, 1996), o "latinoamericanocéntrica", o incluso "indigenista" (HOUTART, 2011). En este esfuerzo de diálogo se ubican ciertas perspectivas de las filosofías de la liberación (SCANNONE, 2009), la traducción intercultural (SANTOS, 2009) y la filosofía intercultural (FORNET BETANCOURT, 2004). También se elaboran formas concretas de diálogo "intercultural" como el discurso del Buen Vivir que toma forma en los planes nacionales de Bolivia y Ecuador (GUDYNAS, 2011; HOUTART, 2011; HUANACUNI, 2010; BELING; GÓMEZ LECHAPTOIS; VANHULST, 2013).

El Buen Vivir o Vivir Bien aúna una serie de propuestas que emergen como reacción y alternativa a los conceptos convencionales sobre el desarrollo, y constituye una experiencia muy interesante y fructífera para responder a las preguntas sobre las formas en que los saberes hegemónicos y no hegemónicos se articulan o no en la esfera de las policies. En general, el Buen Vivir puede ser entendido como una reelaboración de concepciones indígenas desde la modernidad contemporánea, que se halla en pleno proceso de construcción, y que combina principios éticos de la cultura andina, aportes contemporáneos de ciertas corrientes intelectuales contra-hegemónicas y su incorporación a la esfera política en algunos países de América Latina, en particular Ecuador y Bolivia (BELING; GÓMEZ LECHAPTOIS; VANHULST, 2013). Desde estas propuestas se cuestiona "la racionalidad del desarrollo actual, su énfasis en los aspectos económicos y el mercado, su obsesión con el consumo, o el mito de un progreso continuado" (GUDYNAS, 2011, p. 2). En ellas es posible identificar distintos niveles para abordar su construcción: las ideas, los discursos y las prácticas. En el primer plano se encuentran los cuestionamientos radicales a las bases conceptuales del desarrollo, especialmente su apego a la ideología del progreso; en el segundo se halla la crítica a los discursos y las legitimaciones de dichas ideas, y la validación de otras formas de hablar, escribir o pensar nuestro mundo; en el tercero, se encuentran las acciones concretas, tales como proyectos políticos, planes gubernamentales, marcos normativos y formas de elaboración de alternativas al desarrollo convencional (GUDYNAS, 2011).

En este último nivel se encuentran el Plan Nacional para el Buen Vivir de Ecuador 2009-2013 (SENPLADES, 2009), el Plan Nacional de Desarrollo de Bolivia 2006-2010 (BOLIVIA, 2006) y el Plan Nacional de Desarrollo 2010-2015 del programa del MAS, en el mismo país (MAS-IPSP, 2009). En el marco de dichos planes se busca poner en acción la noción del Buen Vivir, incorporando a la acción política pública no sólo los saberes occidentales sino también y principalmente aquellos saberes de los propios pueblos que habitan históricamente el territorio. De esta forma, se promueven iniciativas como el "Taller sobre saberes ancestrales y políticas públicas en la región" organizado por el gobierno de Ecuador en conjunto con Unesco para diversos países de la región, y donde, en este caso particular, el objetivo era dar cuenta de las políticas de gobierno relacionadas con ciencia y tecnología que se estuvieran llevando a cabo en los respectivos países, haciendo especial hincapié en aquellas que incluyen los saberes ancestrales (UNESCO; SENECYT, 2013). 
Más allá de las críticas que puedan hacérsele a dichas propuestas ${ }^{8}$, el Buen Vivir y su implementación en planes nacionales constituye un referente importante dentro del pensar latinoamericano para construir nuevas alternativas de política pública que no se asienten sólo en la primacía de la racionalidad tecnocientífica sino que se construyan en diálogo con otros saberes y prácticas que han sido invisibilizados o excluidos. De ahí la importancia de hacer referencia a esta experiencia que abre posibilidades concretas de integración entre saberes distintos; teniendo siempre en cuenta que la recuperación de saberes subordinados no se reduce a cambiar pensadores europeos por pensadores latinoamericanos, sino que asume la relevancia que tienen las prácticas sociales como espacios de construcción de conocimiento, poniendo al centro aquellos "saberes divergentes que se organizan en torno a registros prácticos y a criterios materiales, en franca oposición a la erudición y al formalismo de los saberes en cada caso hegémonicos" (RIVERA CUSICANQUI; MARGETIC, s.f.).

\section{Trabajo Social y descolonización de las políticas públicas, algunos desafíos a la disciplina}

Así como para otras disciplinas de las ciencias sociales, también para el Trabajo Social constituye un desafío luchar "por construir voces y paradigmas teóricos independientes, en un entorno que no facilita la emergencia de aproximaciones alternativas", tomando en cuenta la preeminencia del pensamiento europeo y estadounidense evidenciada por el Informe Mundial sobre las Ciencias Sociales de Unesco (2010). Es decir, se abre el desafío de dejar de ser sólo "hermeneutas de obras ajenas" y profundizar en la producción del conocimiento propio. Este desafío es particularmente importante en uno de los principales ámbitos de intervención e investigación de la disciplina, como son las políticas públicas. Ello, considerando la estrecha relación de la profesión con el Estado y sus acciones públicas y considerando también la vinculación cotidiana de las y los trabajadores sociales con los "públicos" de las políticas.

Si las políticas públicas han sido sustentadas por una racionalidad reducida, donde el poder de la toma de decisiones queda en manos de un grupo de expertos que presumiría conocer cómo funciona la sociedad, llegando incluso a presumir del conocimiento de las necesidades y deseos humanos, sobre todo las de los grupos menos visibles e influenciables, constituye un desafío para el Trabajo Social cuestionar la hegemonía de aquellos que sustentan su saber en criterios de validez establecidos por y para sí mismos; desafio que se acrecienta si tomamos en cuenta que en general, la profesión se forma y ejerce en dos espacios clave de hegemonía de las formas modernas de racionalidad: la universidad y el Estado. Ni la política, entendida como el ejercicio del poder, ni lo público, entendido como un "espacio colectivo en que se discuten los aspectos que influyen a la vida en sociedad" (DÁVILA; SOTO, 2011, p. 12), pueden ser reducidos a un solo tipo de racionalidad que excluye otras formas de saber y estar en el mundo y por tanto desconoce su rica diversidad.

En este contexto cobra sentido el proceso de descolonización de las políticas públicas que, entre otros elementos, implica recuperar aquello que Rivera y Margetic (s.f.) - con Foucault -, identifican como "saberes sometidos", es decir, "el saber del enfermo, del enfermero, del delincuente", el saber del "usuario", del "beneficiario", del "cliente", del "sujeto", agregaríamos. La recuperación y visibilización de dichos saberes es condición de posibilidad para su real y efectiva puesta en diálogo con los saberes hegemónicos, tal como lo hacen, por ejemplo, las propuestas del Buen Vivir. Para esta tarea se puede echar mano de la larga tradición de pensamiento que toma a América Latina como lugar de enunciación y que también ha sido invisibilizado o menospreciado frente al poderío anglosajón, en el caso de las políticas públicas.

En este sentido, el Trabajo Social y su acción en este ámbito, puede y debe ser repensado desde una pluri-versalidad epistemológica (WALSH, 2007) que tenga en cuenta y dialogue con otras formas de producción de conocimientos que no necesariamente se generan en ámbitos académicos o científicos; para lo cual se hace necesario poner en cuestión las propias bases de la política pública, refutando los presupuestos que localizan la producción de conocimiento únicamente en los "grupos de expertos". Porque cuando habla el rico "lo escuchan en silencio, y ponen por las nubes su talento'; Habla el pobre y dicen: ¿quién es éste?, y si cae encima lo empujan" (DUSSEL, 2004, p. 170). Se trata recuperar el habla del pobre y bajar de las nubes el talento del rico.

\section{Referencia}

BELING, A. E.; GÓMEZ LECHAPTOIS, F.; VANHULST, J. Del Sumak Kawsay al buen vivir: la filosofía andina como base para una racionalidad ambiental moderna. En: GÓMEZ, C. M. (Ed.). La religión en la sociedad post-secular. Bogotá: Universidad Colegio Mayor de Nuestra Señora del Rosario, 2013.

BILBAO, F. El Congreso Americano. Iniciativa de la América. Idea de un congreso federal de las repúblicas [1856]. Disponible en: <www.franciscobilbao.cl>. Acceso en: 10 ago. 2013. 
BOLIVIA. Ministerio de Planificación del Desarrollo. Plan Nacional de Desarrollo 2006-2010. Bolivia digna, soberana, productiva y democrática para Vivir Bien. La Paz: Ministerio de Planificación del Desarrollo, 2006.

CALLON, M. The Role of Lay People in the Production and Dissemination of Scientific Knowledge. Science, Technology and Society. SAGE Journals, v. 4, n. 1, p. 81-94, 1999.

CHAQUÉS BONAFONT, L. Redes de políticas públicas. Madrid: Centro de Investigaciones Sociológicas, 2004.

DÁVILA, M.; SOTO, X. ¿De qué se habla cuándo se habla de políticas públicas? Estado de la discusión y actores en el Chile del bicentenario. Revista Chilena de Administración Pública. Santiago, n. 17, p. 5-33, 2011.

DEVÉS, E. El pensamiento latinoamericano en el siglo XX: entre la modernización y la identidad. Buenos Aires: Biblos, 2000.

DUSSEL, E. Hacia una filosofía política crítica. Bilbao: Desclee de Brouwer S.A., 2001.

. La introducción de la transformación de la filosofía de K.O. Apel y la filosofía de la liberación. En: DUSSEL, E.; APEL, K-O.

Ética del discurso y ética de la liberación. Madrid: Trotta, 2004, p. 73-126.

Filosofía de la cultura y la liberación. México: Universidad Autónoma de la Ciudad de México, 2006.

FORNET BETANCOURT, R. Crítica intercultural de la filosofía latinoamericana actual. Madrid: Editorial Trotta, 2004.

GARCÍA MÁRQUEZ, G. Discurso de aceptación del Premio Nobel de Literatura, 1982.

GÓMEZ LECHAPTOIS, F. Políticas públicas críticas para y desde América Latina. Revista Política y Cultura. México: UAM Xochimilco, n. 40, p. 79-98, otoño 2013.

GONZÁLEZ CASANOVA, P. Sociología de la explotación. Buenos Aires: Clacso, 2006.

GUDYNAS, E. Buen vivir: germinando alternativas al desarrollo. Quito: ALAI, 2011.

GUTIÉRREZ, G. Hacia una teología de la liberación. Bogotá: Indo-America Press Service, 1971.

HOUTART, F. El concepto de sumak kawsai (buen vivir) y su correspondencia con el bien común de la humanidad. Quito: ALAI, 2011. HUANACUNI MAMANI, F. Buen Vivir/Vivir Bien. Filosofía, políticas, estrategias y experiencias regionales andinas. Lima: Coordinadora Andina de Organizaciones Indígenas - CAOI, 2010.

JOBERT, B. Estado, sociedad y políticas públicas. Santiago: LOM, 2004.

KUSCH, R. América profunda. Buenos Aires: Biblos, 2009.

LARRAÍN, J. Modernidad, razón e identidad en América Latina. Barcelona: Andrés Bello, 1996.

MARTÍ, J. Nuestra América. Caracas: Fundación Biblioteca Ayacucho, 2005.

MAS-IPSP. Bolivia país lider. Programa del MAS-IPSP 2010-2015. La Paz, Bolivia, 2009.

MIRANDA, P. Tensiones estructurales y semánticas en las políticas públicas - una cartografía del caso chileno. Textos \& Contextos. Porto Alegre: Pucrs, v. 10, n. 1, p. 137-156, jan./jul. 2011.

MORANDÉ, P. Cultura y modernización en América Latina: ensayo sociológico acerca de la crisis del desarrollismo y de su superación. Santiago: Instituto de Sociología Pontificia Universidad Católica de Chile, 1984.

OSMAN, F. A. Public Policy Making: Theories and their Implications in Developing Countries. Asian Affairs, p. 37-53, 2002.

QUIJANO, A. Colonialidad y Modernidad/Racionalidad. Perú Indígena, Lima, v. 13, n. 29, p. 11-20, 1992.

El fantasma del desarrollo en América Latina. Revista del CESLA, Polonia, n. 1, p. 39-56, 2000.

RIVERA CUSICANQUI, S.; MARGETIC, A. La intolerancia epistemológica como forma de exclusión del saber. Argetina: Universidad Nacional de Lanús, s.f.

RIVERA CUSICANQUI, S. Ch'ixinakax utxiwa: una reflexión sobre prácticas y discursos descolonizadores. Argentina: Tinta Limón, 2010.

ROTH DEUBEL, A-N. Las políticas públicas y sus principales enfoques analíticos. En: ROTH DEUBEL, A-N. (Ed.). Enfoques para el análisis de políticas públicas. Colombia: Universidad Nacional de Colombia, 2010.

SALADINO, A. Pensamiento latinoamericano del s. XIX. Ciudad de México: UNAM, 2009.

SALAZAR, A. ¿Existe una filosofía de nuestra América? México: Siglo XXI, 1968.

SANTOS, B. de S. Una epistemología del Sur. México: Clacso; Siglo XXI, 2009.

. Epistemologías del Sur. Utopía y Praxis Latinoamericana, Venezuela, año 16, n. 54, p- 17-39, 2011.

SANTOS, J. Conflicto de representaciones. América Latina como lugar para la filosofía. Santiago: Fondo de Cultura Económica, 2010. SCANNONE, J. C. La filosofía de la liberación: historia, características, vigencia actual. Teología y Vida, Chile, v. L, p. 59-73, 2009.

SEGUNDO, J. L. Libertação da teologia. Sao Paulo: Loyola, 1978.

SENPLADES. Plan Nacional para el Buen Vivir 2009-2013: Construyendo un Estado Plurinacional e Intercultural. Quito, Ecuador: Senplades, 2009. Disponible en: 〈http://plan.senplades.gob.ec/>. Acceso en: 9 ago. 2013.

SOBRINO, J. Jesucristo liberador: lectura histórico-teológica de Jesús de Nazaret. Madrid: Trotta, 1991.

TUSSIE, D.; DECIANCIO, M. La construcción del saber académico en América Latina: ¿voz de los excluidos o sostén de la tecnocracia? En: ROJAS ARAVENA, F.; ÁLVAREZ-MARÍN, A. (Ed.). América Latina y el Caribe: Globalización y conocimiento. Repensar las Ciencias Sociales. Montevideo: Unesco; Flacso, p. 93-116, 2011.

UNESCO. Informe Mundial sobre las Ciencias Sociales. Las brechas del conocimiento. México: Unesco, 2010.

UNESCO; SENECYT. Taller Incorporando Saberes Diversos a las Políticas de Ciencia, Tecnología e Innovación - Experiencias de América Latina. Quito, 2013. Disponible en: <http://portal.unesco.org/geography/es/ev.php-URL_ID=16636\&URL_DO=DO_ TOPIC\&URL_SECTION=201.html>. Acceso en: 10 ago. 2013. 
WALLERSTEIN, I. El eurocentrismo y sus avatares: los dilemas de las ciencias sociales. En: Mignolo, W. (Comp.). Capitalismo y geopolítica del Conocimiento. El eurocentrismo y la filosofia de la liberación en el debate intelectual contemporaneo. Buenos Aires: Ediciones del Signo, p. 95-116, 1996.

WALSH, C. ¿Son posibles unas ciencias sociales/culturales otras? Reflexiones en torno a las epistemologías decoloniales. Nómadas, Colombia: Universidad Central, n. 26, p. 102-113, 2007.

ZEA, L. El pensamiento latinoamericano. Barcelona: Ariel, 1976.

\section{Notas}

1 U “occidentalocéntrico" si se reconoce la posterior incorporación de la hegemonía del pensamiento estadounidense (SANTOS, 2010).

2 Articulo vinculado al proyecto Fondecyt n. 1110428.

3 El Buen Vivir constituye un nuevo discurso que resulta de la combinación entre los principios éticos de la cultura andina, aportes contemporáneos de ciertas corrientes intelectuales contra-hegemónicas y su reciente incorporación a la esfera política en algunos países de América Latina, en particular Ecuador y Bolivia (BELING; GÓMEZLECHAPTOIS; VANHULST, 2013). Conceptualmente, puede ser entendido como "vivir en plenitud, saber vivir en armonía con los ciclos de la Madre Tierra, del cosmos, de la vida y de la historia, y en equilibrio con toda forma de existencia en permanente respeto" (HUANACUNI MAMANI, 2010, p. 32).

4 A pesar de que el surgimiento de la noción de desarrollo se ubica generalmente en el período que sucede a la Segunda Guerra Mundial, ella aparece como deudora de conceptos anteriores como son el de progreso, civilización, evolución, riqueza y crecimiento. La noción de desarrollo se separa con claridad de estos conceptos a partir del discurso pronunciado por Harry Truman al asumir la presidencia de Estados Unidos, donde se alude a la existencia de amplias regiones del planeta que corresponden a países "subdesarrollados", y se plantea la lucha contra esta situación por parte de los países "desarrollados", en el marco del combate al comunismo.

5 Tal como señala SANTOS (2009), al basarse las prácticas sociales en conocimientos, son a la vez prácticas de saber, es decir, constituyen saberes aplicados.

6 Piénsese aquí el rol o lugar que tienen los saberes no expertos en los modelos predominantes de análisis de políticas públicas en América Latina. Gómez Lechaptois (2013).

7 Estas preguntas forman parte del proyecto de investigación "Saberes expertos y saberes legos en Política Pública en América Latina: un estudio del caso de pobreza, infancia y migración en Chile" presentado durante el año 2013 al Fondo Nacional de Desarrollo Científico y Tecnológico (Chile), por el investigador Patricio Miranda, y las co-investigadoras Francisca Gómez Lechaptois, Claudia Silva y Gabriela Rubilar.

8 Como aquellas que hablan de una integración a-problemática de la cosmovisión aymara al PND, desde donde se podría correr el riesgo de generar simplificaciones como pensar al "ayllu“" como granja colectiva, o al indígena como un proletario; o aquellas que podríamos denominar como 'esencialistas', y que indican que la cosmovisión indígena es irreductible a la perspectiva del desarrollo propuesta por dicho plan por ser una entidad extraña a éste (BELING, GÓMEZ LECHAPTOIS; VANHULST, 2013).

\section{Francisca Gómez Lechaptois}

fgomez@codeni.cl

Magister en Estudios Sociales y Políticos Latinoamericanos

Jefa del Departamento de Estudios de La Fundación Defensa del Niño (CODENI)

Profesora de la Escuela de Trabajo Social de la Pontificia Universidad Católica de Chile (UC) y de la Universidad Alberto Hurtado (UAH)

\section{Fundación Defensa del Niño (CODENI)}

Paseo Presidente Errázuriz Echaurren, 2631

Providencia

Santiago, Chile

\section{Pontificia Universidad Católica de Chile}

Vicuña Mackenna, 4860

San Joaquín

Santiago, Chile

\section{Universidad Alberto Hurtado}

Almirante Barroso, 10

Santiago, Chile 